\title{
ON THE FUNCTIONAL EQUATION OF THE NORMALIZED SHINTANI L-FUNCTION OF SEVERAL VARIABLES
}

\author{
MINORU HIROSE, NOBUO SATO
}

\begin{abstract}
In this paper, we introduce the normalized Shintani L-function of several variables by an integral representation and prove its functional equation. The Shintani L-function is a generalization to several variables of the Hurwitz-Lerch zeta function and the functional equation given in this paper is a generalization of the functional equation of Hurwitz-Lerch zeta function. In addition to the functional equation, we give special values of the normalized Shintani L-function at non-positive integers and some positive integers.
\end{abstract}

\section{INTRODUCTION}

The Shintani zeta functions and the Shintani L-functions are originally introduced by Takuro Shintani in his study of Hecke L-functions of totally real algebraic number fields [6]. He gave, for example, special values of Hecke L-functions at non-positive integers, by representing a Hecke L-function as a linear combination of the Shintani zeta functions. Shintani's original zeta and L-functions are of one variable, but in this paper we consider Shintani L-functions of several variables (see [2]), since sometimes they have more refined information.

Functional equations for zeta and L-functions of one variable are classically known, for example, for Hecke L-functions [1] which are further developed in the Iwasawa-Tate theory [7. Functional equations for zeta and L-functions of several variables are known, for example, for those associated to prehomogeneous vector spaces [5]. However, such functional equations for the Shintani zeta functions or the Shintani L-functions have not been known in general except for the case of the Hurwitz-Lerch zeta function [3] and the case of the double zeta function[4. In this paper, we show that the normalized Shintani L-functions generally satisfy nice functional equations, which is a generalization of the functional equation of the Hurwitz-Lerch zeta function. The functional equation of the normalized Shintani L-function seems applicable to refine the functional equations of Hecke L-functions of totally real number fields.

First of all, we give a definition (Definition 11) of the Shintani L-function by a multiple Dirichlet series. Then, we give an integral representation of the Shintani L-function (Proposition 2) by which we extend the definition of the Shintani Lfunction. We then use a similar integral representation to define the normalized Shintani L-function (Definition 3). This extension of the definition is necessary to 
formulate the functional equation. Throughout this paper, we use the notations

$$
\begin{aligned}
e(z) & =e^{2 \pi i z}, \\
\Gamma_{\mathbb{R}}(s) & =\pi^{-\frac{s}{2}} \Gamma\left(\frac{s}{2}\right), \\
\Gamma_{\mathbb{C}}(s) & =2(2 \pi)^{-s} \Gamma(s),
\end{aligned}
$$

and for $u=\left(u_{1}, \ldots, u_{r}\right), v=\left(v_{1}, \ldots, v_{r}\right) \in \mathbb{R}^{r}$ and $s=\left(s_{1}, \ldots, s_{r}\right) \in \mathbb{C}^{r}$,

$$
\begin{aligned}
u v & =u_{1} v_{1}+\cdots+u_{r} v_{r}, \\
u^{s} & =u_{1}^{s_{1}} \cdots u_{r}^{s_{r}}, \\
1-u & =\left(1-u_{1}, \ldots, 1-u_{r}\right),
\end{aligned}
$$

for simplicity. We also use the notation $(0,1)$ for the unit open interval.

Definition 1. For $s=\left(s_{\nu}\right)_{\nu=1}^{r} \in \mathbb{C}^{r}$ with $\Re\left(s_{1}+\cdots+s_{r}\right)>r, A=\left(a_{\nu \mu}\right)_{\nu, \mu=1}^{r} \in$ $M_{r}\left(\mathbb{R}_{>0}\right)$, and $x=\left(x_{\nu}\right)_{\nu=1}^{r}, y=\left(y_{\mu}\right)_{\mu=1}^{r} \in(0,1)^{r}$ define the Shintani L-function $L(s, A, x, y)$ of order $r$ by

$$
\begin{aligned}
L(s, A, x, y) & =\sum_{0 \leq m_{1}, \cdots, m_{r}} \frac{e\left(m_{1} y_{1}+\cdots+m_{r} y_{r}\right)}{\prod_{\nu=1}^{r}\left(\sum_{\mu=1}^{r} a_{\nu \mu}\left(x_{\mu}+m_{\mu}\right)\right)^{s_{\nu}}} \\
& =\sum_{0 \leq m} \frac{e(m y)}{(A(x+m))^{s}},
\end{aligned}
$$

It is easy to check that the series on the right-hand side converges absolutely in the half space $\Re\left(s_{1}+\cdots+s_{r}\right)>r$ and conditionally for $\Re\left(s_{1}+\cdots+s_{r}\right)>0$.

$L(s, A, x, y)$ has an integral representation which extends the domain of definition of $A$ to $G L_{r}(\mathbb{R})$. This expression is very important in our context, since we cannot avoid $A$ with negative entries for our purpose. Put, for simplicity,

$$
\begin{aligned}
\varphi(t, x, y) & =\frac{e(i t x)}{1-e(y+i t)} \\
F(t, x, y) & =\prod_{\nu=1}^{r} \varphi\left(t_{\nu}, x_{\nu}, y_{\nu}\right) .
\end{aligned}
$$

Then we have the following integral representation for $L(s, A, x, y)$.

Proposition 2. For $\Re\left(s_{1}\right), \ldots, \Re\left(s_{r}\right)>0$, the Shintani L-function $L(s, A, x, y)$ of degree $r$ has the following integral representation.

$$
L(s, A, x, y)=\frac{2^{r}}{\Gamma_{\mathbb{C}}\left(s_{1}\right) \cdots \Gamma_{\mathbb{C}}\left(s_{r}\right)} \int_{0}^{\infty} \cdots \int_{0}^{\infty} F(t A, x, y) t^{s} \frac{d t_{1}}{t_{1}} \cdots \frac{d t_{r}}{t_{r}}
$$

The proof is easy. Note again that, since the integral on the left hand side of Proposition 2 is defined for $A \in G L_{r}(\mathbb{R})$, we extend the definition of $L(s, A, x, y)$ by this integral representation. Next we introduce the normalized Shintani Lfunctions. For a map $\chi:\{1, \cdots, r\} \rightarrow\{0,1\}$, set the quasi-character $|\cdot|_{\chi}^{s}$ on $\left(\mathbb{R}^{r}\right)^{\times}$ by

$$
|t|_{\chi}^{s}=\prod_{\nu=1}^{r} \operatorname{sgn}\left(t_{\nu}\right)^{\chi(\nu)}\left|t_{\nu}\right|^{s_{\nu}}
$$

where sgn denote the signum function on $\mathbb{R}^{\times}$. Then the normalized Shintani Lfunction attached to $\chi$ is defined as follows. 
Definition 3. For a map $\chi:\{1, \cdots, r\} \rightarrow\{0,1\}$, define the normalized Shintani L-function of parity type $\chi$ of degree $r$ by

$$
L_{\chi}(s, A, x, y)=\frac{1}{\Gamma_{\mathbb{C}}\left(s_{1}\right) \cdots \Gamma_{\mathbb{C}}\left(s_{r}\right)} \int_{\left(\mathbb{R}^{r}\right) \times} F(t A, x, y)|t|_{\chi}^{s} \frac{d t_{1}}{t_{1}} \cdots \frac{d t_{r}}{t_{r}},
$$

and its completion by

$$
\hat{L}_{\chi}(s, A, x, y)=|\operatorname{det}(A)|^{\frac{1}{2}} \Gamma_{\chi}(s) L_{\chi}(s, A, x, y)
$$

where we set its gamma factor by $\Gamma_{\chi}(s)=\prod_{\nu=1}^{r} \Gamma_{\mathbb{R}}\left(s_{\nu}+\chi(\nu)\right)$.

The notion of parity type of the normalized Shintani L-function corresponds to the infinity type of the Hecke L-function of a totally real field. As easily seen, by dividing the domain of integration, the normalized Shintani L-function is related to the ordinary Shintani L-function by

$$
L_{\chi}(s, A, x, y)=2^{-r} \sum_{\sigma \in\{ \pm 1\}^{r}} \sigma^{1-\chi} L(s, \sigma A, x, y),
$$

where for $\sigma=\left(\sigma_{\nu}\right)_{\nu=1}^{r} \in\{ \pm 1\}^{r}$, we write $\sigma^{1-\chi}=\prod_{\nu=1}^{r} \sigma_{\nu}^{1-\chi(\nu)}$ and $\sigma A=$ $\left(\sigma_{\nu} a_{\nu \mu}\right)_{\nu, \mu=1}^{r}$. The inversion formula is simply

$$
L(s, A, x, y)=\sum_{\chi} L_{\chi}(s, A, x, y)
$$

where $\sum_{\chi}$ means the sum over all the parity types of degree $r$. Thus, a normalized Shintani L-function is expressible by a finite sum of Shintani L-functions and vica versa, but the normalized Shintani L-function satisfy much simpler functional equations.

Now the main theorem.

Theorem 4. Let $\hat{L}_{\chi}(s, A, x, y)$ be a complete normalized Shintani $L$-function of degree $r$ and parity type $\chi$ defined on $s \in \mathbb{C}^{r}$. Set $i_{\chi}=i^{\sum_{\nu=1}^{r} \chi(\nu)}$. Then $\hat{L}_{\chi}(s, A, x, y)$ satisfies the following functional equation.

$$
\hat{L}_{\chi}(s, A, x, y)=i_{\chi} e(-x y) \hat{L}_{\chi}\left(1-s, A^{*}, y, 1-x\right) .
$$

Here, $A^{*}=\left(A^{t}\right)^{-1}$, the inverse transpose of $A$.

\section{A PRoOf OF The FUnCTIONAL EQUATION}

In this section, we give a proof of Theorem 4. Our proof make use of the (inverse) Fourier transform of $F(t A, x, y)$ and Tate's local functional equation.

Lemma 5. We have

$$
\int_{\mathbb{R}^{r}} F(t A, x, y) e(t k) d t=\frac{i^{r}}{|\operatorname{det} A|} e(-y x) F\left(k A^{*}, y, 1-x\right) .
$$

Proof. Put $u=t A$ and $h=k A^{*}$, then $d t=|\operatorname{det} A|^{-1} d u$ and $t k=u h$. Thus we have

$$
\begin{aligned}
\int_{\mathbb{R}^{r}} F(t A, x, y) e(t k) d t & =\frac{1}{|\operatorname{det} A|} \int_{\mathbb{R}^{r}} F(u, x, y) e(u h) d u \\
& =\frac{1}{|\operatorname{det} A|} \prod_{i=1}^{r} \int_{\mathbb{R}} \varphi\left(u_{i}, x_{i}, y_{i}\right) e\left(u_{i} h_{i}\right) d u_{i},
\end{aligned}
$$


by which we reduce the proof to one variable case. For $\lambda \in \mathbb{R}_{>0}$, consider the positively oriented rectangular contour $C_{\lambda}$ consisting of the intervals $I_{0, \lambda}=[-\lambda, \lambda]$, $I_{1, \lambda}=[\lambda, \lambda+i], I_{2, \lambda}=[-\lambda+i, \lambda+i]$ and $I_{3, \lambda}=[-\lambda,-\lambda+i]$. Then we have

$$
\int_{I_{2, \lambda}} \varphi(u, x, y) e(u h) d u=-e(-x+i h) \int_{I_{0, \lambda}} \varphi(u, x, y) e(u h) d u
$$

so that

$$
\begin{aligned}
(1-e(-x+i h)) \int_{I_{0, \lambda}}+\int_{I_{1, \lambda}}+\int_{I_{3, \lambda}} & =\int_{C_{\lambda}} \\
& =i e(-y(x-i h)) .
\end{aligned}
$$

Thus, by letting $\lambda \rightarrow+\infty$ here, we have

$$
\begin{aligned}
\int_{\mathbb{R}} \varphi(u, x, y) e(u h) d u & =\frac{i e(-y(x-i h))}{1-e(-(x-i h))} \\
& =i e(-y x) \varphi(h, y, 1-x)
\end{aligned}
$$

from which we obtain Lemma 5,

Now, we recall Tate's local functional equation for $\mathbb{R}$.

Lemma 6. Let $\Phi \in \mathcal{S}\left(\mathbb{R}^{r}\right)$ and $\hat{\Phi} \in \mathcal{S}\left(\mathbb{R}^{r}\right)$ be the inverse Fourier transform of $\Phi$. Then, for $0<\Re s_{1}, \ldots, \Re s_{r}<1$, we have

$$
\frac{\int_{\left(\mathbb{R}^{r}\right) \times} \Phi(t)|t|_{\chi}^{s} d^{\times} t}{\Gamma_{\chi}(s)}=i_{\chi}^{-1} \frac{\int_{\left(\mathbb{R}^{r}\right) \times} \hat{\Phi}(t)|t|_{\chi}^{1-s} d^{\times} t}{\Gamma_{\chi}(1-s)}
$$

where $d^{\times} t=\prod_{i=1}^{r}\left|t_{i}\right|^{-1} d t_{i}$ is a Haar measure of $\left(\mathbb{R}^{r}\right)^{\times}$.

Since $\mathcal{S}(\mathbb{R})^{\otimes r}$ is dense in $\mathcal{S}\left(\mathbb{R}^{r}\right)$, Lemma 6 immediately follows from Tate's local functional equation. Now, by a simple calculation, we see that

$$
\begin{aligned}
|\operatorname{det}(A)|^{-\frac{1}{2}} \hat{L}_{\chi}(s, A, x, y) & =\frac{\Gamma_{\chi}(s)}{\prod_{i=1}^{r} \Gamma_{\mathbb{C}}\left(s_{i}\right)} \int_{\left(\mathbb{R}^{r}\right)^{\times}} F(t A, x, y)|t|_{\chi}^{s} \prod_{i=1}^{r} \frac{d t_{i}}{t_{i}} \\
& =\frac{1}{\Gamma_{1-\chi}(s)} \int_{\left(\mathbb{R}^{r}\right)^{\times}} F(t A, x, y)|t|_{1-\chi}^{s} d^{\times} t
\end{aligned}
$$

As it is clear that $F(t A, x, y) \in \mathcal{S}\left(\mathbb{R}^{r}\right)$ for $A \in G L_{r}(\mathbb{R})$ and $x, y \in(0,1)^{r}$, we have by Lemma 5 and Lemma 6

$$
\frac{\int_{\left(\mathbb{R}^{r}\right) \times} F(t A, x, y)|t|_{\chi}^{s} d^{\times} t}{\Gamma_{\chi}(s)}=\frac{i_{1-\chi}}{|\operatorname{det} A|} e(-x y) \frac{\int_{\left(\mathbb{R}^{r}\right) \times} F\left(t A^{*}, y, 1-x\right)|t|_{\chi}^{1-s} d^{\times} t}{\Gamma_{\chi}(1-s)} .
$$

Replacing $\chi$ by $1-\chi$ in the equality above, we obtain the functional equation for $\hat{L}_{\chi}(s, A, x, y)$ for $0<\Re s_{1}, \ldots, \Re s_{r}<1$. Since $\hat{L}_{\chi}(s, A, x, y)$ is a holomorphic function on $\mathbb{C}^{r}$ (see Corollary 12), we can conclude that the functional equation holds for $\mathbb{C}^{r}$.

\section{Several properties of the normalized Shintani L-Function}

In this section, we note several additional properties of the normalized Shintani L-functions. 
Proposition 7. In the case of degree $r=1, L_{\chi}(s, A, x, y)$ admits the following Dirichlet series expressions for $\Re s>1$.

$$
L_{\chi}(s, A, x, y)=\frac{\operatorname{sgn}(A)}{2} \sum_{m \in \mathbb{Z}} \frac{e(m y)}{|A(x+m)|_{\chi}^{s}} .
$$

Proof. We have

$$
L_{\chi}(s, A, x, y)=\frac{1}{2} L(s, A, x, y)-\frac{(-1)^{\chi(1)}}{2} L(s,-A, x, y) .
$$

Since $\varphi(-t A, x, y)=-e(-y) \varphi(t A, 1-x, 1-y)$, we have

$$
\begin{aligned}
L(s,-A, x, y) & =\frac{-e(-y)}{\Gamma_{\mathbb{C}}(s)} \int_{0}^{\infty} \varphi(t A, 1-x, 1-y) d t \\
& =-e(-y) L(s, A, 1-x, 1-y) .
\end{aligned}
$$

Hence, we obtain

$L_{\chi}(s, A, x, y)=\frac{\operatorname{sgn}(A)^{1-\chi(1)}}{2}\left(L(s,|A|, x, y)+(-1)^{\chi(1)} e(-y) L(s,|A|, 1-x, 1-y)\right)$

from which Proposition 7 follows.

From Proposition 7 we see that when $r=1, L_{\chi}(s, A, x, y)$ admits a Dirichlet series expression, which is expressible by a combination of Hurwitz-Lerch zeta functions. However, for $r \geq 2$, we have no Dirichlet series expressions for the normalized Shintani L-functions. This is because we have no Dirichlet series expressions for $L(s, A, x, y)$ when the matrix $A$ has both positive and negative entries. Note that though the integral representation in Proposition 2 is only valid for $x \in(0,1)$, the Dirichlet series expression in Proposition 7 is valid for $x \in \mathbb{R} \backslash \mathbb{Z}$ satisfying the property

$$
L_{\chi}(s, A, x+k, y+l)=e(-k y) L_{\chi}(s, A, x, y)
$$

for $k, l \in \mathbb{Z}$. Moreover, the function $\hat{L}_{\chi}(s, A, x, y)=|\operatorname{det}(A)|^{\frac{1}{2}} \Gamma_{\chi}(s) L_{\chi}(s, A, x, y)$ extended by this formula still satisfy the same functional equation as in Theorem 4. since

$$
\begin{aligned}
\hat{L}_{\chi}(s, A, x, y) & =e(-[x] y) \hat{L}_{\chi}(s, A, x-[x], y-[y]) \\
& =i_{\chi} e(-[x] y-(x-[x])(y-[y])) \hat{L}_{\chi}\left(1-s, A^{*}, y-[y], 1-x+[x]\right) \\
& =i_{\chi} e(-x y) \hat{L}_{\chi}\left(1-s, A^{*}, y, 1-x\right),
\end{aligned}
$$

where $[x]$ denotes the unique integer satisfying $[x] \leq x<[x]+1$. Conversely, we have the following proposition.

Proposition 8. The unique extension of the definition of $L_{\chi}(s, A, x, y)$ to $x, y \in$ $(\mathbb{R} \backslash \mathbb{Z})^{r}$ that satisfy the functional equation

$$
\hat{L}_{\chi}(s, A, x, y)=i_{\chi} e(-x y) \hat{L}_{\chi}\left(1-s, A^{*}, y, 1-x\right),
$$

and the property $L_{\chi}(s, A, x, y+k)=L_{\chi}(s, A, x, y)$, is given by

$$
L_{\chi}(s, A, x, y)=e(-[x] y) L_{\chi}(s, A, x-[x], y-[y]),
$$

where $[x]=\left(\left[x_{1}\right], \ldots,\left[x_{r}\right]\right)$. 
We extend the definition of $L_{\chi}(s, A, x, y)$ quasiperiodically as in Proposition 8 Now, complementarily let us define the functions $G(t, x, y)$ and $R_{\chi}(s, A, x, y)$ by

$$
\begin{aligned}
G(t, x, y) & =e(x y) F(t, x, y) \\
& =\prod_{\nu=1}^{r} \frac{e\left(\left(y_{\nu}+i t_{\nu}\right) x_{\nu}\right)}{\left(1-e\left(y_{\nu}+i t_{\nu}\right)\right)}
\end{aligned}
$$

and

$$
R_{\chi}(s, A, x, y)=e(x y) L_{\chi}(s, A, x, y) .
$$

Then the quasiperiodicity and the functional equation become

$$
\begin{aligned}
& L_{\chi}(s, A, x+k, y+l)=e(-k y) L_{\chi}(s, A, x, y), \\
& R_{\chi}(s, A, x+k, y+l)=e(x l) R_{\chi}(s, A, x, y),
\end{aligned}
$$

for $k, l \in \mathbb{Z}^{r}$, and

$$
\begin{aligned}
& \hat{R}_{\chi}(s, A, x, y)=i_{\chi}^{ \pm 1} \hat{L}_{\chi}\left(1-s, A^{*}, \pm y, \mp x\right), \\
& \hat{L}_{\chi}(s, A, x, y)=i_{\chi}^{ \pm 1} \hat{R}_{\chi}\left(1-s, A^{*}, \pm y, \mp x\right),
\end{aligned}
$$

with $\hat{R}_{\chi}(s, A, x, y)=\Gamma_{\chi}(s) R_{\chi}(s, A, x, y)$.

The following proposition gives a differential property of $L_{\chi}(s, A, x, y)$ and $R_{\chi}(s, A, x, y)$.

Proposition 9. Let $A=\left(a_{\mu \nu}\right)_{\mu, \nu=1}^{r}, A^{*}=\left(a_{\mu \nu}^{*}\right)_{\mu, \nu=1}^{r}$. Then we have

$$
\begin{aligned}
& \frac{\partial}{\partial x_{\nu}} L_{\chi}(s, A, x, y)=-\sum_{\mu=1}^{r} a_{\mu \nu} s_{\mu} L_{\chi+1_{\mu}}\left(s+1_{\mu}, A, x, y\right), \\
& \frac{\partial}{\partial y_{\nu}} R_{\chi}(s, A, x, y)=2 \pi i \sum_{\mu=1}^{r} a_{\mu \nu}^{*} R_{\chi+1_{\mu}}\left(s-1_{\mu}, A, x, y\right),
\end{aligned}
$$

or equivalently,

$$
\begin{aligned}
& \left(\sum_{\nu=1}^{r} a_{\mu \nu}^{*} \frac{\partial}{\partial x_{\nu}}\right) L_{\chi}(s, A, x, y)=-s_{\mu} L_{\chi+1_{\mu}}\left(s+1_{\mu}, A, x, y\right), \\
& \left(\sum_{\nu=1}^{r} a_{\mu \nu} \frac{\partial}{\partial y_{\nu}}\right) R_{\chi}(s, A, x, y)=2 \pi i R_{\chi+1_{\mu}}\left(s-1_{\mu}, A, x, y\right) .
\end{aligned}
$$

Here, we identify a parity type $\chi$ with the vector $\chi=(\chi(\nu))_{\nu=1}^{r} \in(\mathbb{Z} / 2 \mathbb{Z})^{r}$ and we denote by $1_{\mu}=(0, \ldots, 1, \ldots, 0)$ a vector whose entries are 0 except for the $\mu$-th entry being 1.

Proposition 9 follows from the observation

$$
\begin{aligned}
& \frac{\partial}{\partial x_{\nu}} F(t, x, y)=-2 \pi t_{\nu} F(t, x, y), \\
& \frac{\partial}{\partial y_{\nu}} G(t, x, y)=-i \frac{\partial}{\partial t_{\nu}} G(t, x, y),
\end{aligned}
$$

and integration by parts. Using these differential operators, we now see that the functional equation of one parity type can generate the functional equation of any parity type.

Finally we give special values of $L_{\chi}(s, A, x, y)$ at non-positive integers and at some positive integers. Let us first define the multiple Bernoulli-like number $B_{k}(A, x, y)$. 
Definition 10. For $k \in \mathbb{Z}_{>0}^{r}$, we define $B_{k}(A, x, y)$ by the coefficients of the Taylor expansion of $F(t A, x, y)$ i.e.

$$
F(t A, x, y)=\sum_{k \in \mathbb{Z}_{\geq 0}^{r}} B_{k}(A, x, y) \frac{(-2 \pi t)^{k}}{k !},
$$

where for multi-index $k=\left(k_{1}, \ldots, k_{r}\right)$, we put $t^{k}=\prod_{\nu=1}^{r} t_{\nu}^{k_{\nu}}$ and $k !=\prod_{\nu=1}^{r} k_{\nu} !$.

Then we have the following proposition.

Proposition 11. For $k \in \mathbb{Z}_{\geq 0}^{r}$, we have

$$
L_{\chi}(-k, A, x, y)= \begin{cases}B_{k}(A, x, y) & \text { if } k \equiv 1-\chi \quad\left(\bmod 2 \mathbb{Z}^{r}\right) \\ 0 & \text { otherwise }\end{cases}
$$

and for $k \in \mathbb{Z}_{>0}^{r}$ such that $k \equiv \chi\left(\bmod 2 \mathbb{Z}^{r}\right)$,

$$
L_{\chi}(k, A, x, y)=|\operatorname{det}(A)|^{-1} e(-x y) B_{k-1}\left(A^{*}, y, 1-x\right) \frac{(2 \pi i)^{k}}{2^{r}(k-1) !} .
$$

Proof. As one can easily check, the Shintani L-function is expressed in terms of the contour integral

$$
\prod_{\nu=1}^{r}\left(\frac{2}{\Gamma_{\mathbb{C}}\left(s_{\nu}\right)\left(e\left(s_{\nu}\right)-1\right)}\right) \int_{L} \cdots \int_{L} F(t A, x, y) t^{s} \frac{d t_{1}}{t_{1}} \cdots \frac{d t_{r}}{t_{r}},
$$

where $L$ is a contour which starts and ends at $+\infty$ and circles the origin once counterclockwise without encircling any poles other than the origin. By the formula $\Gamma_{\mathbb{C}}(s) \Gamma_{\mathbb{C}}(1-s)=4 i(e(s / 2)-e(-s / 2))^{-1}$, we see that

$$
L(s, A, x, y)=\prod_{\nu=1}^{r}\left(\frac{e\left(-\frac{s_{\nu}}{2}\right) \Gamma_{\mathbb{C}}\left(1-s_{\nu}\right)}{2 i}\right) \int_{L} \cdots \int_{L} F(t A, x, y) t^{s} \frac{d t_{1}}{t_{1}} \cdots \frac{d t_{r}}{t_{r}} .
$$

From the definition of $B_{k}(A, x, y)$ and the residue theorem, we find that, for $k \in$ $\mathbb{Z}_{\geq 0}^{r}$

$$
\begin{aligned}
L(-k, A, x, y) & =(2 \pi i)^{r} \prod_{\nu=1}^{r}\left(\frac{e\left(\frac{k_{\nu}}{2}\right) \Gamma_{\mathbb{C}}\left(1+k_{\nu}\right)}{2 i}\right) B_{k}(A, x, y) \frac{(-2 \pi)^{k}}{k !} \\
& =B_{k}(A, x, y) .
\end{aligned}
$$

Using the relation $L_{\chi}(s, A, x, y)=2^{-r} \sum_{\sigma \in\{ \pm 1\}^{r}} \sigma^{1-\chi} L(s, \sigma A, x, y)$, we have

$$
\begin{aligned}
L_{\chi}(-k, A, x, y) & =2^{-r} \sum_{\sigma \in\{ \pm 1\}^{r}} \sigma^{1-\chi} B_{k}(\sigma A, x, y) \\
& =2^{-r} B_{k}(A, x, y) \sum_{\sigma \in\{ \pm 1\}^{r}} \sigma^{1-\chi+k} .
\end{aligned}
$$

Hence we have proved the former statement of the Proposition 11. The latter statement is proved by applying the functional equation for $L_{\chi}(s, A, x, y)$ to the former statement.

From the contour integral representation of $L_{\chi}(s, A, x, y)$ in the proof of Proposition 11, the following fact follows immediately.

Corollary 12. $L_{\chi}(s, A, x, y)$ is a holomorphic function on $s \in \mathbb{C}^{r}$. 


\section{REFERENCES}

[1] E. Hecke, Eine neue Art von Zetafunktionen und ihre Beziehungen zur Verteilung der Primzahlen, Mathematische Zeitschrift, 6(1):11-51, 1920.

[2] H. Hida, Elementary theory of L-functions and Eisenstein series, London Mathematical Society Student Texts, 1993.

[3] M. Lerch, Note sur la fonction $K(w, x, s)=\sum_{k=0}^{\infty} \frac{e(2 k \pi i x)}{(w+k)^{s}}$, Acta Mathematica 11 (1887): $19-24$.

[4] K. Matsumoto, Functional equations for double zeta-functions, Math. Proc. Cambridge Phil. Soc (2004). 136, 1-7.

[5] F. Satō, Zeta functions in several variables associated with prehomogeneous vector spaces. I. Functional equations, Tôhoku Math. J. (2) 34 (1982), no. 3, 437-483.

[6] T. Shintani, On evaluation of zeta functions of totally real algebraic number fields at non positive integers, J. Fac. Sci. Univ. Tokyo Sect. IA Math. 23 (1976), no. 2, 393-417.

[7] J. Tate, Number theoretic background, Proc. Symp. Pure Math. 33, part 2 (1979), 3-26. 